\title{
Clinical Significance of Pretreatment Red Blood Cell Distribution Width as a Predictive Marker for Postoperative Morbidity After Esophagectomy for Esophageal Cancer: A Retrospective Study
}

\author{
Naoya Yoshida, MD, PhD ${ }^{1,2}$, Tomo Horinouchi, $\mathrm{MD}^{1}$, Tasuku Toihata, $\mathrm{MD}^{\mathbf{1}}$, Kazuto Harada, MD, $\mathrm{PhD}^{\mathbf{1}}$, \\ Kojiro Eto, MD, PhD ${ }^{1}$, Hiroshi Sawayama, MD, $\mathbf{P h D}^{1}$, Masaaki Iwatsuki, MD, $\mathbf{P h D}^{1}$, Yohei Nagai, MD, $\mathbf{P h D}^{1}$, \\ Takatsugu Ishimoto, MD, PhD ${ }^{1,2}$, Yoshifumi Baba, MD, PhD ${ }^{1}$, Yuji Miyamoto, MD, PhD $^{1}$, and \\ Hideo Baba, MD, PhD ${ }^{1}$ \\ ${ }^{1}$ Department of Gastroenterological Surgery, Graduate School of Medical Sciences, Kumamoto University, Chuoku, \\ Kumamoto, Japan; ${ }^{2}$ Division of Translational Research and Advanced Treatment Against Gastrointestinal Cancer, \\ Kumamoto University, Chuoku, Kumamoto, Japan
}

\begin{abstract}
Background. Clinical significance of red blood cell distribution (RDW) as a predictive marker for the incidence of postoperative morbidity after esophagectomy for esophageal cancer has not been established.

Methods. This study included 634 consecutive patients who underwent three-incisional esophagectomy with lymphadenectomy for esophageal cancer between April 2005 and November 2020. Correlation between pretreatment RDW and patient background, cancer background, and short-term outcome after esophagectomy were retrospectively investigated.

Results. Eighty patients (12.6\%) had a high pretreatment RDW (>14.2), which correlated with malnutrition estimated by body mass index, hemoglobin, total lymphocyte count, albumin, and total cholesterol. High pretreatment RDW was an independent risk factor for postoperative severe morbidity of grade IIIb or higher based on the Clavien-Dindo classification (hazard ratio [HR] 3.90, 95\% confidence interval $[\mathrm{CI}]$ 1.707-8.887; $p=0.0012$ ) and reoperation (HR 4.39, 95\% CI 1.552-12.390; $p=0.0053$ ) after open esophagectomy (OE). However, RDW was not
\end{abstract}

(C) Society of Surgical Oncology 2021

First Received: 28 April 2021

Accepted: 11 August 2021;

Published Online: 1 September 2021

H. Baba, MD, PhD

e-mail: hdobaba@kumamoto-u.ac.jp associated with postoperative morbidity incidence after minimally invasive esophagectomy (MIE).

Conclusions. Pretreatment RDW may be a surrogate marker for nutritional status and could be a predictive marker for postoperative severe morbidity, reoperation, and possibly pneumonia after OE. On the contrary, the lower invasiveness of MIE may have reduced the effect of pretreatment malnutrition on morbidity incidence, which could explain the insignificant relationship between RDW and poor short-term outcomes in MIE.

Esophagectomy for esophageal cancer is among the most invasive surgeries associated with frequent postoperative morbidity compared with other gastrointestinal cancer surgeries. The preoperative prediction of the incidence of postoperative morbidity is considered important because of its prophylactic value and the possible reduction of surgery-related mortality. Several predictive markers for postoperative morbidity have been previously reported. ${ }^{1-3}$ Notably, measurable markers based on common blood tests are useful because these could be performed at any institute and be objectively evaluated. To date, the mean corpuscular volume (MCV) and several nutritional markers are suggested as useful predictors of postoperative morbidity after esophagectomy. ${ }^{4-6}$

Red blood cell distribution width (RDW) is a parameter in red blood cell size variability and is used for estimating the pathogenesis of anemia. ${ }^{7}$ High RDW may also be associated with several cardiovascular diseases ${ }^{8-10}$ and poor prognosis of inflammatory diseases. ${ }^{11-15}$ Recently, the 
correlation between elevated RDW and high mortality in patients with coronavirus disease 2019 (COVID-19) has attracted increasing attention. ${ }^{16}$ However, the mechanism underlying the association of RDW with the incidence and prognoses of these diseases remains unclear.

RDW has been considered an indicator of inflammation, malnutrition, microvascular disorder, oxidative stress, and prothrombotic effect. ${ }^{17}$ Although the progression of these conditions could be a risk for postoperative morbidities after highly invasive surgery, there have been no studies regarding the effect of RDW on short-term outcomes after gastroenterological surgeries. Thus, this study aimed to clarify the correlation between pretreatment RDW and the short-term outcomes after esophagectomy for esophageal cancer. Moreover, the correlation between pretreatment RDW and patient and cancer backgrounds was also investigated to elucidate how high RDW reflects the frequent incidence of postoperative morbidities.

\section{MATERIAL AND METHODS}

\section{Patients}

A total of 803 consecutive three-incisional esophagectomies with lymphadenectomy were performed for esophageal cancer at the Kumamoto University Hospital between April 2005 and November 2020. Of these, 24 cases of two-stage esophagectomies, 43 cases of salvage esophagectomies after definitive chemoradiotherapy (CRT), and 102 cases with insufficient clinical data (70 data points for alcohol status, 1 for smoking status, 12 for pretreatment data of total cholesterol, 9 for RDW, 6 for C-reactive protein [CRP], and 4 for total lymphocyte count) were excluded. Eventually, 634 patients were enrolled in this study (Fig. 1). RDW examined in this study

803 three-incisional esophagectomy for esophageal cancer (2005.4-2020.11)

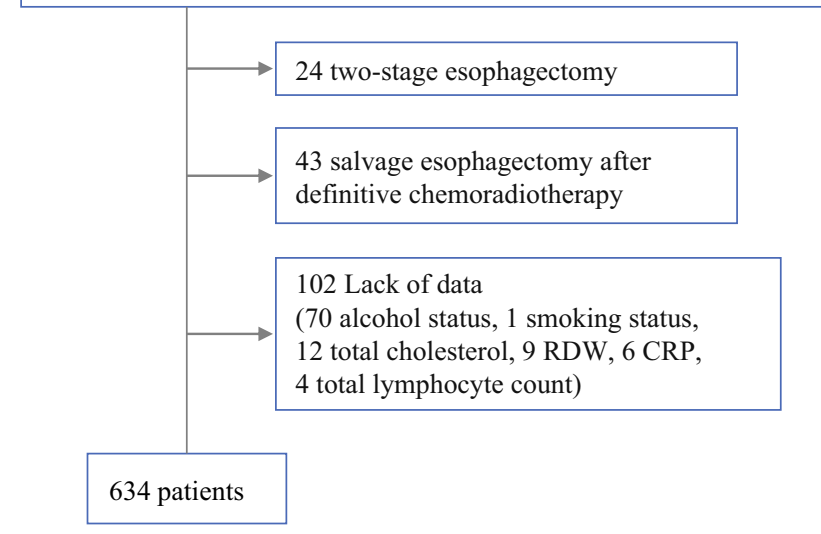

FIG. 1 Flow chart of analyzed patients. $R D W$ red blood cell distribution width, $C R P$ C-reactive protein was measured before the administration of any treatments. Patients were divided into two groups according to the institutional standard value of pretreatment RDW-high $(14.2<)$ and normal $(\leq 14.2)$. A retrospective investigation for the association between RDW and clinicopathological features, blood tests, and short-term outcomes after surgery was performed using a prospectively entered institutional clinical database. This study was conducted in accordance with the ethical standards of the Helsinki Declaration of 1975. The institutional Ethics Committee approved the study procedures (registry number 2193). Written informed consent was waived for the patients because of the retrospective nature of this study.

\section{Treatment Strategy}

Treatment strategy details have been previously described. $^{4}$ For non-T4 node-positive tumors, neoadjuvant chemotherapy has been administered since August 2008, whereas for T4 tumors, neoadjuvant CRT has been generally performed. The Union for International Cancer Control TNM staging (version 7) was used to classify the pretreatment clinical stage. ${ }^{18}$

\section{Surgery}

Esophagectomy was defined as three-incisional (in the neck, chest, and abdomen) esophagectomy with lymphadenectomy. The extent of lymphadenectomy was determined in accordance with the 2012 guidelines formulated by the Japan Esophageal Society. ${ }^{19}$ Regarding manipulation in the thorax, open esophagectomy (OE) was performed before April 2011. Minimally invasive esophagectomy (MIE) for clinical stage T1 and T2 cancers was initiated in May 2011. With progress in the operator's and team's skills, we have routinely performed MIE for cancers of all stages since September 2011. During MIE, chest manipulation was performed from the right thorax in the left semi-prone position.

\section{Perioperative Management}

Details regarding perioperative management have been previously described. ${ }^{20}$ Bolus administration of methylprednisolone and continuous intravenous administration of neutrophil elastase inhibitors for $24 \mathrm{~h}$ were routinely conducted at the start of surgery. Moreover, precautionary antibiotics were administered every $4 \mathrm{~h}$ during surgery. Extubation was performed in the operating room shortly after surgery, and enteral nutrition was generally started on the first day after surgery. 


\section{Definitions of Morbidities}

The morbidity details have been previously described. ${ }^{5}$ Severe morbidity was defined as a complication of grades IIIb or higher according to the Clavien-Dindo classification (CDc) system. ${ }^{21}$ Initial ventilatory support for $>48 \mathrm{~h}$ or reintubation for respiratory failure, need for tracheostomy, and pneumonia were defined as respiratory morbidities. Moreover, any respiratory morbidity requiring intervention or surgical treatment was included under this definition.

\section{Statistical Methods}

Statistical analyses were performed using the StatView $^{\mathrm{TM}}$ version 5.0 software package (Abacus Concepts, Inc., Berkeley, CA, USA). The Chi-square test and MannWhitney $U$ test were used to compare groups and unpaired samples, respectively. Fisher's exact test was used for the comparisons of groups containing a matrix with fewer than five patients. Data on pretreatment blood tests were divided into two groups according to the institutional standard value. Logistic regression analysis was performed to estimate the hazard ratio (HR) with a $95 \%$ confidence interval (CI) for each morbidity. The following data were adopted to analyze independent risk factors for the incidence of severe morbidity, reoperation, and pneumonia among patients who underwent OE: age (per 10 years), sex (male vs. female), body mass index (BMI; $<18.5 \mathrm{vs.} \geq 18.5 \mathrm{~kg} /$ $\mathrm{m}^{2}$ ), performance status (PS; 0 vs. 1 and 2), American Society of Anesthesiologists physical status (ASAPS; 1 and 2 vs. 3), Brinkman index (tobacco number/day $\times$ year, for a 100-point increase), chronic obstructive pulmonary disease (COPD; yes vs. no), neoadjuvant treatment (chemotherapy and CRT vs. none), dissection field ( $\leq 2$ vs. 3 ), conduit (stomach vs. others), operative time (per $60 \mathrm{~min}$ increase), blood loss (per $100 \mathrm{~g}$ increase), clinical stage (II, III, and IV vs. I), and RDW (>14.2 vs. $\leq 14.2$ ). Factors with a probability level of $\leq 0.1$ were adopted for subsequent multivariate analyses. Variables with a $p$ value $<0.05$ were presumed to be independent risk factors.

\section{RESULTS}

\section{Association Between Pretreatment Red Blood Cell Distribution Width and Clinicopathological Features}

Among all patients, $80(12.6 \%)$ had a high pretreatment RDW (>14.2). High RDW significantly correlated with lower BMI $(p=0.045)$; however, high RDW was not related to any other clinicopathological factors, such as age, sex, past smoking and drinking, PS, ASAPS, comorbidity, clinical stage, and histological type of cancer
(Table 1). The blood test revealed that high RDW was significantly associated with lower hemoglobin $(p<0.0001)$ and total lymphocyte count $(p=0.012)$. Moreover, high RDW exhibited a trend toward lower serum albumin and total cholesterol. Nevertheless, high RDW was also irrelevant to inflammation, as suggested by white blood cell count and CRP (Table 2).

\section{Short-Term Outcomes After Surgery}

Because the distribution of OE and MIE was considerably different between the high and normal RDW groups, the short-term outcomes for OE and MIE were separately investigated. Table 3 shows the short-term outcomes in patients who underwent $\mathrm{OE}$, according to pretreatment RDW. Operative time and blood loss were significantly higher in the high RDW group, possibly due to frequent use of the colon conduit (high RDW, 19\%; normal RDW, 6\%; $p=0.0046$ ). Moreover, postoperative severe morbidity, pneumonia, and reoperation were significantly frequent in the high RDW group. The association of high RDW with frequent severe morbidity, reoperation, and pneumonia was also seen in patients who underwent reconstruction using a gastric conduit (electronic supplementary Table 1). By contrast, short-term outcomes in patients who underwent MIE were statistically equivalent between the high and normal RDW groups (Table 4).

\section{Risk Factors for Postoperative Morbidities of Grades IIIb or Higher According to the Clavien-Dindo Classification, Reoperation, and Pneumonia in Patients who Underwent Open Esophagectomy}

Table 5 shows the results of multivariate analyses for postoperative morbidities in patients who underwent OE. High pretreatment RDW was an independent risk factor for postoperative severe morbidity of CDc grade IIIb or higher (HR 3.90, 95\% CI 1.707-8.887; $p=0.0012$ ) and reoperation (HR 4.39, 95\% CI 1.552-12.390; $p=0.0053$ ). It also exhibited a trend toward frequent incidence of pneumonia, although it was not statistically significant (HR 2.70, 95\% CI $0.995-7.351 ; p=0.051)$.

\section{DISCUSSION}

In this study, several interesting results were obtained for the clinical value of pretreatment RDW in esophagectomy for esophageal cancer. First, high RDW may be a surrogate marker for malnutrition estimated by BMI, hemoglobin, total lymphocyte count, albumin, and total cholesterol. Second, high RDW was not relevant to factors other than nutrition, such as age, sex, past smoking and 
TABLE 1 Association between pretreatment red blood cell distribution width and patient characteristics

\begin{tabular}{|c|c|c|c|c|}
\hline \multirow[t]{2}{*}{ Clinical, epidemiological, and pathological feature } & \multirow[t]{2}{*}{ Total $N$} & \multicolumn{2}{|c|}{ Pretreatment red blood cell distribution } & \multirow[t]{2}{*}{$p$ value } \\
\hline & & $\leq 14.2$ & $14.2<$ & \\
\hline All cases & 634 & 554 & 80 & \\
\hline Age, years $[$ mean $\pm \mathrm{SD}]$ & $66.5 \pm 8.3$ & $66.7 \pm 8.2$ & $65.2 \pm 8.7$ & 0.14 \\
\hline Male patients & $556(88)$ & $483(87)$ & $73(91)$ & 0.30 \\
\hline Body mass index $[$ mean $\pm \mathrm{SD}]$ & $21.9 \pm 3.1$ & $22.0 \pm 3.1$ & $21.3 \pm 3.1$ & 0.045 \\
\hline Estimated ethanol consumption $^{\mathrm{a}}[$ mean $\pm \mathrm{SD}]$ & $2450 \pm 2040$ & $2450 \pm 2060$ & $2450 \pm 1900$ & 0.98 \\
\hline Brinkman index ${ }^{\mathrm{b}}[$ mean $\pm \mathrm{SD}]$ & $770 \pm 570$ & $770 \pm 580$ & $800 \pm 460$ & 0.64 \\
\hline Performance status & & & & 0.82 \\
\hline 0 & $565(89)$ & $495(89)$ & $70(88)$ & \\
\hline 1 & $64(10)$ & $55(10)$ & $9(11)$ & \\
\hline 2 & $5(1)$ & $4(1)$ & $1(1)$ & \\
\hline ASA physical status & & & & 0.24 \\
\hline 1 & $125(20)$ & $111(20)$ & $14(18)$ & \\
\hline 2 & $483(76)$ & $423(76)$ & $60(75)$ & \\
\hline 3 & $26(4)$ & $20(4)$ & $6(8)$ & \\
\hline \multicolumn{5}{|l|}{ Comorbidity } \\
\hline Diabetes mellitus & $133(21)$ & $117(21)$ & $16(20)$ & 0.82 \\
\hline Chronic obstructive pulmonary disease & $196(31)$ & $167(30)$ & $29(36)$ & 0.27 \\
\hline Cardiovascular & $334(53)$ & $295(53)$ & $39(49)$ & 0.45 \\
\hline Clinical stage & & & & 0.25 \\
\hline $0, \mathrm{I}$ & $276(44)$ & $248(45)$ & $28(35)$ & \\
\hline II & $113(18)$ & $94(17)$ & $19(24)$ & \\
\hline III & $204(32)$ & $178(32)$ & $26(33)$ & \\
\hline IV & $41(6)$ & $34(6)$ & $7(9)$ & \\
\hline Pathology & & & & 0.70 \\
\hline Squamous cell carcinoma & $577(91)$ & $505(91)$ & $72(90)$ & \\
\hline Adenocarcinoma & $36(6)$ & $30(5)$ & $6(8)$ & \\
\hline Others & $21(3)$ & $19(3)$ & $2(3)$ & \\
\hline Neoadjuvant treatment & & & & 0.83 \\
\hline None & $359(57)$ & $315(57)$ & $44(55)$ & \\
\hline Chemotherapy & $208(33)$ & $182(33)$ & $26(33)$ & \\
\hline Chemoradiotherapy & $67(11)$ & $57(10)$ & $10(13)$ & \\
\hline Surgical procedure, MIE & $355(56)$ & $318(57)$ & $37(46)$ & 0.060 \\
\hline
\end{tabular}

Data are expressed as $n(\%)$ unless otherwise specified

MIE minimally invasive esophagectomy, $S D$ standard deviation, ASA American Society of Anesthesiologists

${ }^{a}$ Ethanol consumption was calculated as follows: estimated daily ethanol consumption $[(0.4 \times$ whisky $+0.2 \times$ distilled spirit $+0.15 \times$ wine and sake $+0.04 \times$ beer $) \times$ year]

${ }^{\mathrm{b}}$ Brinkman index was calculated as follows: number of cigarettes/day $\times$ smoking duration (year)

drinking, comorbidities, cancer stage, and histological type. Third, high RDW was an independent risk factor for severe morbidity, reoperation, and possibly pneumonia after OE, but not after MIE.

RDW is an indicator of variation in red blood cell size. In patients with anemia, RDW increases because of the mixture of different erythrocyte sizes via erythrocyte turnover. In patients with chronic anemia, the size of erythrocytes is uniform, irrespective of macrocytic or microcytic anemia; hence, RDW becomes normalized. RDW also reflects bone marrow function and is used in estimating the pathogenesis of anemia. ${ }^{7} \mathrm{RDW}$ could also reflect inflammation and may be a predictive marker for activity level and survival in several inflammatory diseases, such as inflammatory bowel disease, ${ }^{11}$ chronic hepatitis, ${ }^{12}$ $\mathrm{COPD},{ }^{13}$ acute pancreatitis, ${ }^{14}$ acute respiratory distress 
TABLE 2 Association between pretreatment red blood cell distribution width and blood parameter test results

\begin{tabular}{|c|c|c|c|c|}
\hline \multirow[t]{2}{*}{ Blood test } & \multirow[t]{2}{*}{ Total $N$} & \multicolumn{2}{|c|}{ Pretreatment red blood cell distribution } & \multirow[t]{2}{*}{$p$ value } \\
\hline & & $\leq 14.2$ & $14.2<$ & \\
\hline All cases & 634 & 554 & 80 & \\
\hline Hemoglobin, g/dL [mean \pm SD] & $13.6 \pm 1.5$ & $13.8 \pm 1.4$ & $12.7 \pm 1.7$ & $<0.0001$ \\
\hline Hemoglobin, $<11.6 \mathrm{~g} / \mathrm{dL}$ & $50(8 \%)$ & $33(6 \%)$ & $17(21 \%)$ & $<0.0001$ \\
\hline White blood cell count, $/ \mu \mathrm{L}[$ mean $\pm \mathrm{SD}]$ & $6490 \pm 2740$ & $6540 \pm 2830$ & $6110 \pm 1940$ & 0.19 \\
\hline White blood cell count, $<3300 / \mu \mathrm{L}$ & $11(2 \%)$ & $10(2 \%)$ & $1(1 \%)$ & $>0.99$ \\
\hline Total lymphocyte count, $/ \mu \mathrm{L}[$ mean $\pm \mathrm{SD}]$ & $1740 \pm 630$ & $1770 \pm 640$ & $1580 \pm 550$ & 0.012 \\
\hline Total lymphocyte count, $<1600 / \mu \mathrm{L}$ & $282(44 \%)$ & $229(43 \%)$ & $43(54 \%)$ & 0.074 \\
\hline C-reactive protein, $\mathrm{mg} / \mathrm{dL}[$ mean $\pm \mathrm{SD}]$ & $0.45 \pm 0.97$ & $0.47 \pm 1.02$ & $0.32 \pm 0.44$ & 0.21 \\
\hline C-reactive protein, $\geq 1.00 \mathrm{mg} / \mathrm{dL}$ & $72(11 \%)$ & $67(12 \%)$ & $5(6 \%)$ & 0.12 \\
\hline Serum albumin, g/dL [mean $\pm \mathrm{SD}]$ & $4.04 \pm 0.39$ & $4.05 \pm 0.39$ & $3.97 \pm 0.39$ & 0.074 \\
\hline Serum albumin, $<3.5 \mathrm{~g} / \mathrm{dL}$ & $45(7 \%)$ & $38(7 \%)$ & $7(9 \%)$ & 0.54 \\
\hline Total cholesterol, mg/dL $[$ mean $\pm \mathrm{SD}]$ & $197 \pm 35$ & $197 \pm 35$ & $193 \pm 39$ & 0.33 \\
\hline Total cholesterol, $<180 \mathrm{~g} / \mathrm{dL}$ & $212(33 \%)$ & $178(32 \%)$ & $34(43 \%)$ & 0.071 \\
\hline
\end{tabular}

$S D$ standard deviation

TABLE 3 Pretreatment red blood cell distribution width, surgical feature, and short-term surgical outcomes of patients who underwent open esophagectomy

\begin{tabular}{|c|c|c|c|c|}
\hline \multirow{2}{*}{ Surgical data and morbidities } & \multirow[t]{2}{*}{ Total $N$} & \multicolumn{2}{|c|}{ Pretreatment red blood cell distribution } & \multirow[t]{2}{*}{$p$ value } \\
\hline & & $\leq 14.2$ & $14.2<$ & \\
\hline All cases & 279 & 236 & 43 & \\
\hline Operation time, min $[$ mean $\pm \mathrm{SD}]$ & $540 \pm 110$ & $540 \pm 110$ & $570 \pm 100$ & 0.034 \\
\hline Blood loss, $\mathrm{g}[$ mean $\pm \mathrm{SD}]$ & $580 \pm 440$ & $560 \pm 380$ & $740 \pm 670$ & 0.012 \\
\hline \multicolumn{5}{|l|}{ Postoperative morbidity } \\
\hline Any morbidity, CDc grade II or higher & $111(40)$ & $89(38)$ & $22(51)$ & 0.097 \\
\hline Severe morbidity, CDc grade IIIb or higher & $33(12)$ & $20(8)$ & $13(30)$ & $<0.0001$ \\
\hline Respiratory morbidity & $48(17)$ & $37(16)$ & $11(26)$ & 0.11 \\
\hline Pneumonia & $21(8)$ & $14(6)$ & $7(16)$ & 0.018 \\
\hline Cardiovascular morbidity & $14(5)$ & $13(6)$ & $1(2)$ & 0.70 \\
\hline Leak & $32(11)$ & $25(11)$ & $7(16)$ & 0.28 \\
\hline Reoperation & $19(7)$ & $10(4)$ & $9(21)$ & $<0.0001$ \\
\hline
\end{tabular}

Data are expressed as $n(\%)$ unless otherwise specified

$S D$ standard deviation, $C D c$ Clavien-Dindo classification

syndrome, ${ }^{15}$ and several cardiovascular diseases. ${ }^{8-10}$ Recently, several studies on the significance of RDW as a predictive marker for COVID-19 mortality have been attracting increasing attention. ${ }^{16}$ Moreover, RDW could be a prognostic marker for several malignancies, including esophageal cancer. ${ }^{22,23}$ However, the mechanism behind the significant association between high RDW and the prognosis of the aforementioned diseases has not been established.
To date, no previous studies regarding the association of pretreatment RDW and short-term outcomes after gastroenterological surgery are available. To the best of our knowledge, this is the first study to disclose that high RDW may correlate with the incidence of postoperative morbidity after esophagectomy. In this study, RDW seemed a mere surrogate marker of nutritional status. Although a huge cohort study suggested that smoking habit may affect RDW level, ${ }^{24}$ no such associations were observed in this study. Other factors that could affect the incidence of 
TABLE 4 Pretreatment red blood cell distribution width, surgical features, and short-term surgical outcomes of patients who underwent minimally invasive esophagectomy

\begin{tabular}{|c|c|c|c|c|}
\hline \multirow[t]{2}{*}{ Surgical data and morbidities } & \multirow[t]{2}{*}{ Total $N$} & \multicolumn{2}{|c|}{ Pretreatment red blood cell distribution } & \multirow[t]{2}{*}{$p$ value } \\
\hline & & $\leq 14.2$ & $14.2<$ & \\
\hline All cases & 355 & 318 & 37 & \\
\hline Operation time, min $[$ mean $\pm \mathrm{SD}]$ & $580 \pm 100$ & $580 \pm 100$ & $570 \pm 100$ & 0.54 \\
\hline Blood loss, g [mean \pm SD] & $230 \pm 290$ & $240 \pm 300$ & $190 \pm 230$ & 0.36 \\
\hline \multicolumn{5}{|l|}{ Postoperative morbidity } \\
\hline Any morbidity, CDc grade II or higher & $121(34)$ & $108(34)$ & $13(35)$ & 0.89 \\
\hline Severe morbidity, CDc grade IIIb or higher & $46(13)$ & $42(13)$ & $4(11)$ & 0.80 \\
\hline Pulmonary morbidity & $49(14)$ & $44(14)$ & $5(14)$ & 0.96 \\
\hline Pneumonia & $31(9)$ & $29(9)$ & $2(5)$ & 0.76 \\
\hline Cardiovascular morbidity & $26(7)$ & $26(8)$ & 0 & 0.092 \\
\hline Leak & $46(13)$ & $40(13)$ & $6(16)$ & 0.53 \\
\hline Reoperation & $24(7)$ & $22(7)$ & $2(5)$ & $>0.99$ \\
\hline
\end{tabular}

Data are expressed as $n(\%)$ unless otherwise specified

$S D$ standard deviation, $C D c$ Clavien-Dindo classification

TABLE 5 Logistic regression analysis (backward stepwise elimination) for morbidities of grades IIIb or higher according to the Clavien-Dindo classification, reoperation, and pneumonia in patients who underwent open esophagectomy

\begin{tabular}{|c|c|c|c|c|c|}
\hline \multirow[t]{2}{*}{ Morbidity } & \multirow[t]{2}{*}{ Characteristics } & \multicolumn{2}{|l|}{ Univariate analysis } & \multicolumn{2}{|l|}{ Multivariate analysis } \\
\hline & & HR $(95 \% \mathrm{CI})$ & $p$ value & HR $(95 \%$ CI $)$ & $p$ value \\
\hline \multirow[t]{4}{*}{$\mathrm{CDc}$ grade IIIb or higher } & Dissection field $\leq 2$ (vs. 3 ) & $0.42(0.157-1.139)$ & 0.089 & $0.45(0.159-1.262)$ & 0.13 \\
\hline & Conduit stomach (vs. others) & $0.24(0.090-0.646)$ & 0.0046 & $0.33(0.103-1.061)$ & 0.063 \\
\hline & Operation time (for $60 \mathrm{~min}$ increase) & $1.17(0.979-1.401)$ & 0.085 & $1.03(0.823-1.277)$ & 0.82 \\
\hline & Red blood cell distribution $14.2<($ vs. $\leq 14.2)$ & $4.68(2.111-10.373)$ & 0.0001 & $3.90(1.707-8.887)$ & 0.0012 \\
\hline \multirow[t]{4}{*}{ Reoperation } & Conduit stomach (vs. others) & $0.11(0.036-0.305)$ & $<0.0001$ & $0.17(0.047-0.590)$ & 0.0055 \\
\hline & Operation time (for $60 \mathrm{~min}$ increase) & $1.28(1.031-1.589)$ & 0.026 & $1.06(0.803-1.387)$ & 0.70 \\
\hline & Blood loss (for $100 \mathrm{~g}$ increase) & $1.08(0.997-1.179)$ & 0.059 & $1.03(0.934-1.138)$ & 0.54 \\
\hline & Red blood cell distribution $14.2<($ vs. $\leq 14.2)$ & $5.98(2.268-15.78)$ & 0.0003 & $4.39(1.552-12.390)$ & 0.0053 \\
\hline \multirow[t]{2}{*}{ Pneumonia } & Operation time (for $60 \mathrm{~min}$ increase) & $1.17(0.979-1.401)$ & 0.085 & $1.03(0.823-1.277)$ & 0.82 \\
\hline & Red blood cell distribution $14.2<(\mathrm{vs} . \leq 14.2)$ & $3.08(1.165-8.161)$ & 0.023 & $2.70(0.995-7.351)$ & 0.051 \\
\hline
\end{tabular}

$H R$ hazard ratio, $C I$ confidence interval, $C D c$ Clavien-Dindo classification

postoperative morbidities were equivalent between the high and normal RDW groups. The same result was obtained in several studies reporting that pretreatment malnutrition increased postoperative morbidities in highly invasive gastroenterological procedures, such as esophagectomy, ${ }^{25-27}$ pancreatoduodenectomy, ${ }^{28}$ and rectal surgery. ${ }^{29}$

The Controlling Nutritional Status (CONUT) score is one indicator for the nutritional status estimation and could be a predictive marker for postoperative morbidity after several gastroenterological surgeries. ${ }^{5,30,31}$ In this study, the CONUT score in the high RDW group was significantly higher than that in the normal RDW group (high RDW, $1.6 \pm 1.6$; normal RDW, $1.2 \pm 1.3 ; p=0.0096)$. Moreover, the percentage of patients with malnutrition estimated by the CONUT score was significantly higher in the high RDW group (high RDW, 41\%; normal RDW, 32\%; $p=0.025)$ [data not shown]. The Prognostic Nutritional Index (PNI) is the first identified nutrition-related indicator and could predict complications after esophagectomy. 6 In this study, the PNI was also significantly worse in the high RDW group than in the normal RDW group (high RDW, $47.5 \pm 5.1$; normal RDW, $49.3 \pm 5.3 ; p=0.0050$ ) [data 
not shown]. These results support that RDW reflected nutritional status and affected short-term outcomes after esophagectomy.

RDW reportedly increases in several diseases, such as heart failure, atrial fibrillation, peripheral vascular disease, coronary artery disease, stroke, and pulmonary hypertension. ${ }^{8-10} \mathrm{Li}$ et al. have reviewed the effects of high RDW on cardiovascular disease. ${ }^{17}$ This study discussed how high RDW reflected microvascular disorder, high inflammatory cytokine, oxidative stress, and prothrombotic effects. Thus, patients with high RDW may have several latent disadvantages other than malnutrition, which could have influenced the frequent incidence of postoperative morbidities.

$\mathrm{OE}$ is considered more invasive and associated with more frequent postoperative morbidities than MIE. ${ }^{1,32,33}$ No studies have clarified the correlation between preoperative malnutrition and poor short-term outcomes in MIE to date. In general, poor preoperative conditions could severely affect the incidence of postoperative morbidity as the surgery becomes more invasive. The lower invasiveness of MIE may have reduced the effect of pretreatment malnutrition on the incidence of morbidities, which could explain the irrelevance between high RDW and poor shortterm outcomes in MIE.

We previously reported that high MCV was associated with low BMI and higher frequency of habitual alcohol and tobacco use, which significantly increased pulmonary morbidities after esophagectomy. ${ }^{4} \mathrm{We}$ investigated the association of MCV with patient's backgrounds and shortterm outcomes using the current cohort. Consequently, high MCV was correlated with lower BMI $(p=0.040)$ and more frequent habitual alcohol $(p<0.0001)$ and tobacco use $(p=0.0002)$; however, MCV was not related to total lymphocyte count $(p=0.74)$, serum albumin $(p=0.13)$ and cholesterol $(p=0.13)$ levels, and malnutrition in CONUT $(p=0.47)$. Although RDW could reflect malnutrition based on the blood test, CONUT, and PNI, it was not related to habitual alcohol and tobacco use. Thus, we consider that both MCV and RDW reflect different backgrounds. Finally, we conducted multivariate analysis to calculate the incidence of postoperative pneumonia, including both MCV and RDW, as an element. Only RDW was identified as an independent risk factor for pneumonia after OE (HR 2.94, 95\% CI 1.079-7.988; $p=0.035$ ). Based on the result, RDW may be superior to MCV in the prediction of postoperative pneumonia in patients with $\mathrm{OE}$.

Nevertheless, this study has several limitations. First, this was a retrospective study at a single institute and with a comparatively long study period; hence, there could have been historical bias regarding treatment strategy and perioperative management. Second, many patients had to be excluded due to insufficient data, which could be a selection bias.

\section{CONCLUSION}

Pretreatment RDW may be a surrogate marker for nutritional status and could be a predictive marker for postoperative severe morbidity, reoperation, and possibly pneumonia after OE; however, further multi-institutional investigation with a large cohort is necessary to establish the importance of pretreatment RDW for predicting shortterm outcomes after esophagectomy.

Supplementary Information The online version contains supplementary material available at https://doi.org/10.1245/s10434021-10719-2.

DISCLOSURES Naoya Yoshida and Takatsugu Ishimoto are affiliated to a department supported by Chugai Pharmaceutical Co., Ltd and Yakuruto Honsya Co., Ltd, but declare no conflicts of interest in relation to this current research. Tomo Horinouchi, Tasuku Toihata, Kazuto Harada, Kojiro Eto, Hiroshi Sawayama, Masaaki Iwatsuki, Yohei Nagai, Yoshifumi Baba, Yuji Miyamoto, and Hideo Baba have no conflicts of interest or financial ties to disclose regarding this current research.

\section{REFERENCES}

1. Yoshida N, Baba Y, Hiyoshi Y, et al. Duration of smoking cessation and postoperative morbidity after esophagectomy for esophageal cancer: how long should patients stop smoking before surgery? World J Surg. 2016;40:142-7.

2. Yoshida N, Morito A, Nagai Y, et al. Clinical importance of sputum in the respiratory tract as a predictive marker of postoperative morbidity after esophagectomy for esophageal cancer. Ann Surg Oncol. 2019;26:2580-6.

3. Yoshida N, Baba Y, Kuroda D, et al. Clinical utility of exhaled carbon monoxide in assessing preoperative smoking status and risks of postoperative morbidity after esophagectomy. Dis Esophagus. 2018. https://doi.org/10.1093/dote/doy024.

4. Yoshida N, Kosumi K, Tokunaga R, et al. Clinical importance of mean corpuscular volume as a prognostic marker after esophagectomy for esophageal cancer: a retrospective study. Ann Surg. 2020;271:494-501.

5. Yoshida N, Baba Y, Shigaki H, et al. Preoperative nutritional assessment by controlling nutritional status (CONUT) is useful to estimate postoperative morbidity after esophagectomy for esophageal cancer. World J Surg. 2018;40:1910-7.

6. Filip B, Scarpa M, Cavallin F, et al. Postoperative outcome after oesophagectomy for cancer: Nutritional status is the missing ring in the current prognostic scores. Eur $J$ Surg Oncol. 2015;41:787-94.

7. Buttarello M. Laboratory diagnosis of anemia: are the old and new red cell parameters useful in classification and treatment, how? Int J Lab Hematol. 2016;38(Suppl 1):123-32.

8. Parizadeh SM, Jafarzadeh-Esfehani R, Bahreyni A, et al. The diagnostic and prognostic value of red cell distribution width in cardiovascular disease; current status and prospective. Biofactors. 2019;45:507-16. 
9. Pilling LC, Atkins JL, Kuchel GA, Ferrucci L, Melzer D. Red cell distribution width and common disease onsets in 240,477 healthy volunteers followed for up to 9 years. PLoS One. 2018;13(9):e0203504. https://doi.org/10.1371/journal.pone. 0203504.

10. Petrauskas LA, Saketkoo LA, Kazecki T, et al. Use of red cell distribution width in a population at high risk for pulmonary hypertension. Respir Med. 2019;150:131-5.

11. Katsaros M, Paschos P, Giouleme O. Red cell distribution width as a marker of activity in inflammatory bowel disease: a narrative review. Ann Gastroenterol. 2020;33:348-54.

12. Wang J, Huang R, Yan X, et al. Red blood cell distribution width: A promising index for evaluating the severity and long-term prognosis of hepatitis B virus-related diseases. Dig Liver Dis. 2020;52:440-6.

13. Kalemci S, Akin F, Sarihan A, Sahin C, Zeybek A, Yilmaz N. The relationship between hematological parameters and the severity level of chronic obstructive lung disease. Pol Arch Intern Med. 2018;128:171-7.

14. Goyal H, Awad H, Hu ZD. Prognostic value of admission red blood cell distribution width in acute pancreatitis: a systematic review. Ann Transl Med. 2017;5:342.

15. Yu XS, Chen ZQ, Hu YF, et al. Red blood cell distribution width is associated with mortality risk in patients with acute respiratory distress syndrome based on the Berlin definition: a propensity score matched cohort study. Heart Lung. 2020;49:641-5.

16. Foy BH, Carlson JCT, Reinertsen E, et al. Association of red blood cell distribution width with mortality risk in hospitalized adults with SARS-CoV-2 infection. JAMA Netw Open. 2020;3:e2022058.

17. Li N, Zhou H, Tang Q. Red blood cell distribution width: a novel predictive indicator for cardiovascular and cerebrovascular diseases. Dis Markers. 2017;2017:7089493.

18. Sobin LH, Gospodarowicz MK, Wittekind C. International Union against Cancer. In: TNM classification of malignant tumours, 7th ed. Chichester, West Sussex; Hoboken, NJ: Wiley-Blackwell; 2010.

19. Kuwano H, Nishimura Y, Oyama T, et al. Guidelines for diagnosis and treatment of carcinoma of the esophagus April 2012 edited by the Japan Esophageal Society. Esophagus. 2015;12:1-30.

20. Yoshida N, Nakamura K, Kuroda D, et al. Preoperative smoking cessation is integral to the prevention of postoperative morbidities in minimally invasive esophagectomy. World J Surg. 2018;42:2902-9.

21. Dindo D, Demartines N, Clavien PA. Classification of surgical complications: a new proposal with evaluation in a cohort of 6336 patients and results of a survey. Ann Surg. 2004;240:205-13.

22. Hirahara N, Tajima Y, Fujii Y, et al. Prognostic significance of red cell distribution width in esophageal squamous cell carcinoma. J Surg Res. 2018;230:53-60.
23. Wang PF, Song SY, Guo H, Wang TJ, Liu N, Yan CX. Prognostic role of pretreatment red blood cell distribution width in patients with cancer: a meta-analysis of 49 studies. J Cancer. 2019;10:4305-17.

24. Jayasuriya NA, Kjaergaard AD, Pedersen KM, et al. Smoking, blood cells and myeloproliferative neoplasms: meta-analysis and Mendelian randomization of 2.3 million people. Br J Haematol. 2020;189:323-34.

25. Yoshida N, Harada K, Iwatsuki M, Baba Y, Baba H. Precautions for avoiding pulmonary morbidity after esophagectomy. Ann Gastroenterol Surg. 2020;4:480-4.

26. Wang P, Li Y, Sun H, et al. Predictive Value of Body Mass Index for Short-Term Outcomes of Patients with Esophageal Cancer After Esophagectomy: a Meta-analysis. Ann Surg Oncol. 2019;26:2090-103.

27. Papaconstantinou D, Vretakakou K, Paspala A, et al. The impact of preoperative sarcopenia on postoperative complications following esophagectomy for esophageal neoplasia: a systematic review and meta-analysis. Dis Esophagus. 2020. https://doi.org/ 10.1093/dote/doaa002.

28. Kim E, Lee DH, Jang JY. Effects of preoperative malnutrition on postoperative surgical outcomes and quality of life of elderly patients with periampullary neoplasms: a single-center prospective cohort study. Gut Liver. 2019;13:690-7.

29. Wolf JH, Ahuja V, D'Adamo CR, Coleman J, Katlic M, Blumberg D. Preoperative nutritional status predicts major morbidity after primary rectal cancer resection. $J$ Surg Res. 2020;255:325-31.

30. Ahiko Y, Shida D, Horie T, et al. Controlling nutritional status (CONUT) score as a preoperative risk assessment index for older patients with colorectal cancer. BMC Cancer. 2019;19:946.

31. Takagi K, Domagala P, Polak WG, Buettner S, Ijzermans JNM. The controlling nutritional status score and postoperative complication risk in gastrointestinal and hepatopancreatobiliary surgical oncology: a systematic review and meta-analysis. Ann Nutr Metab. 2019;74:303-12.

32. Biere SS, van Berge Henegouwen MI, Maas KW, et al. Minimally invasive versus open oesophagectomy for patients with oesophageal cancer: a multicentre, open-label, randomised controlled trial. Lancet. 2012;379:1887-92.

33. Yoshida N, Yamamoto H, Baba H, et al. Can minimally invasive esophagectomy replace open esophagectomy for esophageal cancer?: latest analysis of 24,233 esophagectomies from the Japanese national clinical database. Ann Surg. 2020;272:118-24.

Publisher's Note Springer Nature remains neutral with regard to jurisdictional claims in published maps and institutional affiliations. 